\title{
AIDS-Related Lymphoblastic Lymphoma
}

National Cancer Institute

\section{Source}

National Cancer Institute. AIDS-Related Lymphoblastic Lymphoma. NCI Thesaurus. Code C8298.

A lymphoblastic lymphoma occurring in HIV-positive patients. 